\title{
Trust Your GuT
}

\author{
Roger Coron, MD, Jennifer Hurd, MD, Steven Ludwin, MD, Patricia Kozuch, MD
}

\section{Case Presentation}

MG is a 56-year-old female with no significant past medical history who presented to TJUH in October 2007 with a two year history of abdominal pain and weight loss. At presentation, she described her pain as non-radiating, 7 out of 10 , gnawing, burning and located in the epigastrum and left upper quadrant. The pain initially occurred approximately 15 minutes postprandially and lasted 30 minutes. Over the two year period, the pain progressed to lasting for hours following meals. Dietary modifications and food consistency did not help the pain. The patient began avoiding oral intake, and her weight declined from 120 to 80 pounds. She denied nausea, vomiting, diarrhea, constipation, hematochezia, melena and change in stool frequency. Over the two years prior to admission the patient had an extensive workup with multiple gastroenterologists. Her initial workup included a colonoscopy, EGD, abdominal ultrasound and computerized tomography (CT) of the abdomen and pelvis. These were all reportedly normal and the patient was diagnosed with irritable bowel syndrome and treated symptomatically with a PPI. Due to lack of improvement, a second gastroenterologist repeated the EGD/colonoscopy and diagnosed her with peptic ulcer disease secondary to multiple gastric ulcers. She was treated with a PPI and carafate. A capsule study showed gastric and jejunal ulcers with cobblestone appearance of the small bowel which led to the diagnosis of Crohn's disease. She was treated briefly with Pentasa. The patient sought care as an outpatient at TJUH Division of Gastroenterology. Mesenteric ischemia was initially high on the differential. An MRA of the abdomen revealed patent mesenteric vessels. Other workup included gastric emptying scan, push enteroscopy, endoscopic ultrasound (EUS), full-thickness biopsy in the operating room, fasting gastrin level, and an NSAID level. These studies were mostly inconclusive. Other studies including an octreotide scan, gastrin levels, and chromogranin A levels were either normal or inconclusive. Furthermore, it was discovered that the gastric $\mathrm{pH}$ was 4.4, arguing against a gastrin producing tumor as seen in Zollinger-Ellison Syndrome.

At the time of admission, her medications were pantoprazole 40 $\mathrm{mg}$ BID and ciprofloxacin $500 \mathrm{mg}$ BID, both of which offered only mild relief. She has a 25 pack year smoking history, does not drink alcohol or use other drugs. Her family history is significant for a brother and father having coronary artery disease. On examination the patient appeared thin and pale. The abdomen was not tender to palpation, however, an abdominal bruit could be auscultated. Admission labs were within normal limits except for a WBC count of 12,000 without a left shift. The question of mesenteric ischemia was again entertained. An abdominal ultrasound with dopplers revealed a greater than $70 \%$ stenosis of the superior mesenteric artery (SMA). CT angiography (CTA) of the abdomen revealed occlusion of the celiac artery, near occlusion of the SMA, and stenosis of the inferior mesenteric artery (IMA). The magnetic resonance angiogram (MRA) done six months earlier was post-processed and finally revealed stenosis of the SMA. Vascular surgery was consulted and a decision was made to pursue traditional angiography and stenting by interventional radiology. Interventional radiology placed 2 overlapping stents in the SMA which subsequently demonstrated excellent filling of the SMA and branches. Since adequate collateral circulation was observed, the celiac artery was not treated. The post-procedure course was complicated by thrombosis of the left brachial artery prompting thrombectomy. Approximately two weeks after her procedure, the patient had no abdominal pain, is eating voraciously and had already gained 5 pounds.

\section{Discussion}

Chronic mesenteric ischemia (CMI) can be distinguished from acute mesenteric ischemia by the time course of the symptoms and etiology of the disease. Acute mesenteric ischemia can be caused by four mechanisms: SMA embolus or thrombosis, acute mesenteric venous thrombosis, and non-occulsive mesenteric ischemia. ${ }^{1}$ The focus of this article will be chronic mesenteric ischemia. Common risk factors include hyperlipidemia, diabetes, and smoking. ${ }^{2}$ Although there are many possible causes of CMI, most commonly it is secondary to atherosclerosis. ${ }^{2}$ The celiac artery (CA) and SMA are the most commonly affected, although the IMA can be involved. Due to the slow development of the stenosis, particularly when atherosclerosis is the etiology, patients may develop collateral circulation to the affected vessels. Often symptoms do not develop until two vessels have become occluded. Symptoms commonly include post-prandial pain beginning 15-60 minutes after food ingestion and last $\sim 1-4$ hours, manifest by sitophobia, weight loss, nausea, and vomiting. Involvement of the CA distribution may also cause gastroparesis, gastric ulceration, and even gallbladder dyskinesia. While both physical exam and common laboratory tests are usually unremarkable, it is common for patients to have cachexia and stigmata of vascular disease including an abdominal bruit. ${ }^{2}$

Traditional angiography has been the gold standard for diagnosis of CMI. Recently, however, duplex ultrasonography (US), CTA, and MRA are increasingly used to evaluate compromise of the mesenteric arteries. Duplex US is more accurate in identifying SMA stenosis $(90 \%)$ in comparison to CA stenosis $(80 \%)$. Areas of stenosis are identified by increases in peak systolic or velocity of blood flow $(C A>200 \mathrm{~cm} / \mathrm{s}$, SMA $>275 \mathrm{~cm} / \mathrm{s}) .^{7}$ Results may be affected by variations of body habitus, bowel gas, respiration, and anatomy. ${ }^{2}$

CTA and MRA are also reliable methods for the diagnosis of CMI. ${ }^{4}$ In the presence of a negative CTA study (particularly the multidetector-row CTA), it is unlikely for the patient to have CMI. MRA can identify greater than $90 \%$ of SMA lesions, $75-$ $90 \%$ of CA lesions, and $25 \%$ of IMA lesions. ${ }^{2}$ Furthermore, some studies have reported $100 \%$ sensitivity of diagnosing 


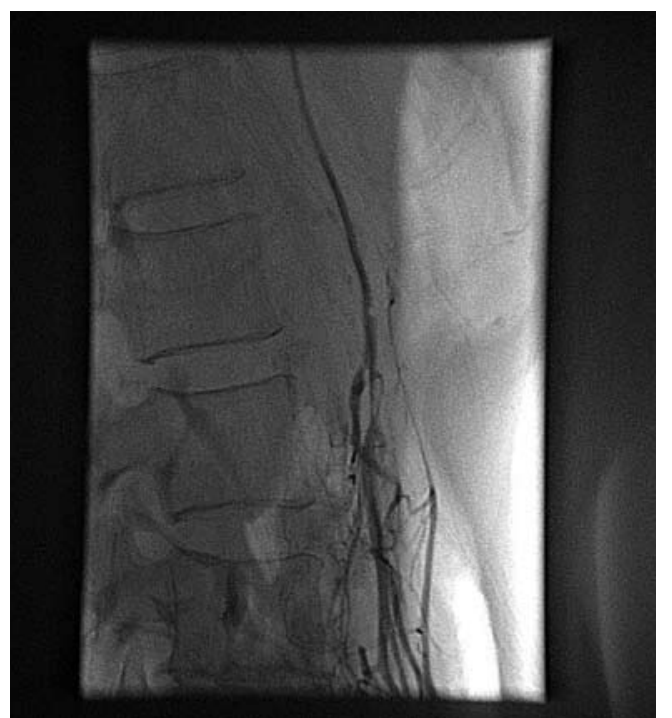

Figure 1. SMA before stent placement.

CA and SMA stenosis when compared to the gold standard of invasive angiography. ${ }^{2}$

When two or more mesenteric vessels are occluded or significantly stenosed in the setting of post-prandial abdominal pain and weight loss, there are two therapeutic options. In the past, surgical revascularization used to be the only option available. This involved transaortic endarterectomy or antegrade vs. retrograde bypass surgery. One study showed a $93 \%$ success rate for endarterectomy with a high patency rate at 1 and 3 years. Unfortunately, many of the patients requiring revascularization are malnourished and at higher risk for perioperative complications. Complication rates range from 15-33\% with mortalitiy up to $17 \% .^{3}$

The other therapeutic option for CMI is percutaneous angioplasty (PCA) with or without stent placement. Unfortunately, it is difficult to compare this modality to surgery. A study involving 25 patients revealed that clinical benefit at 11 months was $91 \%$ and patency of the stents was $92 \% .{ }^{5}$ Furthermore, the mean complication rate for endovascular therapy is $10 \%$ with a mortality rate up to $5 \% .^{3}$ Overall, it appears that endovascular repair may have less associated morbidity and mortality, however surgery offers the benefit of a lower re-stenosis rate. ${ }^{6}$

Finally, it should be emphasized that this case reiterates the importance of clinical history in diagnosis. This patient's diagnosis was initially prolonged despite a fairly classic history, hence CMI was not entertained. However, a false negative MRA further delayed her diagnosis. Furthermore, the authors point out that this patient should never have been diagnosed with irritable bowel syndrome. The diagnosis of IBS can be made using the Rome criteria. Weight loss is a red flag and should have excluded this diagnosis.

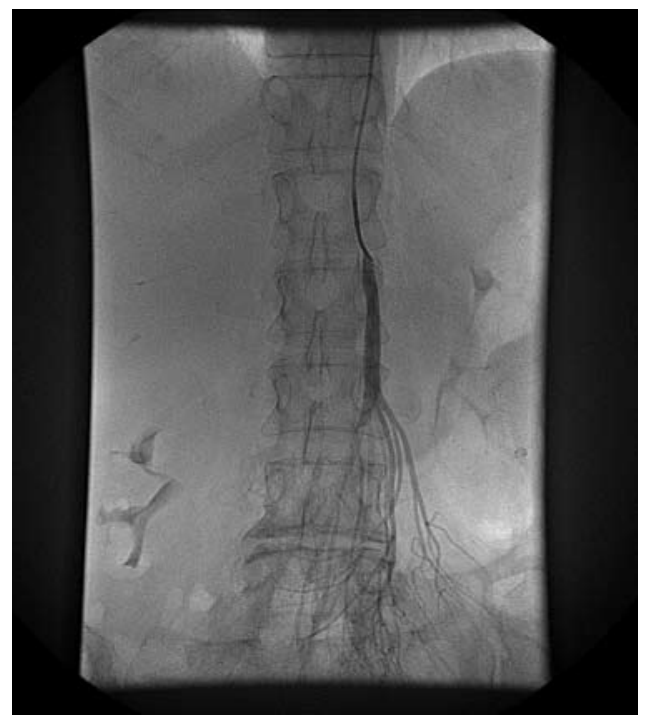

Figure 2. SMA after stent placement.

While imaging and laboratory tests are generally very useful in assisting clinicians make diagnoses, these results should never supercede clinical acumen.

\section{Key Points}

Postprandial abdominal pain and weight loss are the typical symptoms seen in chronic mesenteric ischemia (CMI).

1. Usually, 2 of the 3 major mesenteric vessels must be occluded before the patient develops symptoms.

2. Diagnosis can be made by MRA, US with dopplers, or CTA.

3. Treatment options include vascular surgery and endovascular stenting.

\section{References}

1. Kozuch PL and LJ Brandt. Review article: diagnosis and management of mesenteric ischaemia with an emphasis on pharmacotherapy. Aliment Pharmacol Ther. 2005; 21: 201-215.

2. Sreenarasimhaiah J. Chronic mesenteric ischemia, Best Practice \& Research Clinical Gastroenterology. 2005; 19(2):283-295.

3. Schaefer PJ, Schaefer FK, Mueller-Huelsbeck S, Jahnke T. Chronic mesenteric ischemia: stenting of mesenteric arteries. Abdominal Imaging. 2007; 32(3):304-309.

4. Cognet F, Salem DB, Dranssart M, Cercueil J-P, Weiller M, et al. Chronic Mesenteric Ischemia: Imaging and Percutaneous Treatment. Radiographics. 2002; 22:863-880.

5. Sharafuddin MJ, Olson CH, Sun S, Kresowik TF, Corson JD. Endovascular treatment of celiac and mesenteric arteries stenoses: Applications and results. Journal of Vascular Surgery. 2003; 38:692-698.

6. Kasirajan K, O’Hara PJ, Gray BH, Hertzer NR, Clair DG, et al. Chronic mesenteric ischemia: Open surgery versus percutaneous angioplasty and stenting. Journal of Vascular Surgery. 2001; 33:63-71.

7. Perko MJ. Duplex US for assessment of superior mesenteric artery blood flow. European Journal of Vascular and Endovascular Surgery. 2001; 21:106-117.Silent Assassin: Coronary Artery Disease in A Type II Diabetic Srinath Vemuri, MS IV and Neerav Sheth, MD 